\title{
La recepción de la filosofía analítica en España ${ }^{1}$
}

\author{
JOSÉ LUIS BLASCO \\ Universidad de Valencia
}

Me resulta difícil abordar una cuestión, la recepción de la filosofía analítica en el panorama filosófico español, por varias razones que paso a considerar:

1) En primer lugar, resulta muy complejo delimitar el concepto de filosofia analítica, es un concepto de textura abierta, utilizando un término de Waismann (yo diría incluso muy abierta); que incluye métodos, estilos, enfoques de problemas, preferencias sobre otras cuestiones, etc., pero difícilmente incluye ni un cuerpo de doctrinas ni una nómina de pensadores.

Esta ambigüedad hace difícil el planteamiento del problema ya que si bien todos coincidiremos en que Wittgenstein, Russell, Quine, etc. son clásicos de la filosofía llamada analítica, otros nombres harian dudar a más de un especialista.

2) La irrupción de la filosofía analítica es lenta. No niego que fuera moda en un determinado momento de vida filosófico-política española, sin embargo, antes de esa eclosión por razones diversas habían llegado a nuestro panorama textos importantes; por citar algún ejemplo, Russell, como premio Nobel había tenido traducciones tempranas de sus obras no sólo de filosofía política y social, sino de obras técnicas de filosofía de la matemática y de la lógica. La filosoffa analítica, sersis lato, comienza a introducirse antes del 39 y el trauma de la guerra cortó esta apertura de la filosofía española hasta la decada de los sesenta. A este tema me referiré brevemente después.

3) Es difícil deslindar la lógica actual (llámese matemática, o como se quie- ra) de la filosofía analítica. Aunque técnica y conceptualmente son diferentes y no tienen entre sí más vinculación que la que tienen la lógica y la metafísica en Aristóteles o la lógica y la filosofía trascendental en Kant, el hecho histórico es que la lógica matemática y la filosofía analítica han entrado de la mano en nuestra comunidad filosófica y, además, la lógica y la filosofía analítica, al menos la clásica (Círculo de Viena, Russell...), han crecido juntas.

4) ¿Había una filosofía "española», sobre la que pudiera incidir la «filosofía analitican, más allá de la escolástica oficial del franquismo? Yo diria sin duda que no, aunque volveré sobre el tema, y ello condicionó una recepción esencialmente contradictoria. Dejando de lado la escolástica tradicional y particularmente oficializada a partir del 39 , temo que la filosofía española consiste fundamentalmente en "recepciones» y en este sentido me toca analizar la "recepción" de una filosofía como se podía analizar en el mismo período histórico otras recepciones (existencialismo, marxismo...).

\section{La situación antes de 1939}

Nadie duda de que en el campo de la filosofia, como en la mayoría de áreas científicas y culturales, el trauma de la guerra civil supuso la frustración dura y drástica de su proceso de europeización del pensamiento español. Por lo que a la filosofía analítica se refiere es necesario recordar, aunque sólo sea a modo de ejemplo, la traducción castellana de Joaquín Xirau, en 1928, de Los 
problemas de la filosofía de B. Russell, libro que en una reedición de 1970 , prologada por Emilio Lledó todavía sigue circulando en facultades e incluso en institutos de bachillerato, y la, lamentablemente no reeditada. Introducció a la logística. Amb aplicacions a la filosofia i a les matematiques de David García (léase David García Bacca) publicada en Barcelona por el Institut d'Estudis Catalans en 1934; quizá esta obra hubiera abierto la incorporación de la tradición lógica de Frege y Russell a nuestro pensamiento, y sin embargo, como es sabido, esa apertura no se produce hasta la publicación en 1953 de la Lógica matemática de Ferrater Mora y H. Leblanc (FCE) y posteriomente la publicación de la Introducción a la lógica y al análisis formal de Manuel Sacristán en 1964 (Ariel).

Obviamente, antes del 39 hubo otras referencias bibliográficas del pensamiento analítico, pero como ya he dicho no pretendo hacer aquí un trabajo erudito sino una valoración general . Y en este sentido sí que cabe constatar, quizá, que la apertura del pensamiento español que supuso Ortega y la Revista de Occidente fue poco sensible a estos movimientos de pensamiento que se desarrollaban tanto en la Centroeuropa prenazi como en la Inglaterra de la primera mitad de siglo.

\section{La década de los cuarenta $y$ cincuenta}

Baste una breve nota sobre la filosofía de la postguerra para enmarcar la recepción de la filosofía analítica en los años sesenta. La filosofía sólo tenúa presencia académica en Madrid, Barcelona y Murcia (más tarde a principios de los cincuenta los estudios de Murcia se trasladaron a Valencia). Aquella universidad del final de la década de los cincuenta estaba dominada por el pen- samiento eclesiástico en todas sus facciones y guerrillas: tomistas, agustinianos, suarecianos...; y en aquel contexto no existian ni la lógica formal, ni la filosofía de la ciencia, ni la filosofia del lenguaje, todas ellas disciplinas incorporadas a la académica europea en la primera mitad del siglo $\mathrm{XX}$, ni tan siquiera la teoría del conocimiento, que había tenido su origen académico en la universidad alemana neokantiana de mediados del siglo XIX. Muchos de los que actualmente profesamos la enseñanza y la investigación en filosofía nos "formamos" en aquella filosofía académica esclerotizada y sacralizada.

Fuera de la Academia existían algunos aires de pensamiento fresco, la mayoría en el exilio iberoamericano (no citaré ahora nombres ya que son de todos conocidos) y a los que debemos el único contacto que los estudiantes ${ }^{2}$ podíamos tener, fundamentalmente en las "reboticas" de las librerias. Dentro de la Península nos traia aire europeo la obra de Ortega y el celebrado Naturaleza, Historia, Dios de Zubiri que hablaba de ciencia a la vez que de teología. Pero la filosofía analítica no fue tematizada en este contexto.

Por terminar este pequeño esbozo de panorama de la filosofía académica española respecto a la filosofía analítica, citaré un par de textos como testimonio del "espiritu de la época», Permitame el lector que no los comente, y deje su identificación a la perspicacia del filósofo para así conocer de las décadas a las que me refiero. Un manual universitario, que tuvimos que sufrir, para informar de la lógica matemática decía asî: "En lógica matemática, simbólica o algoritmica, descuella Bertrand Russell, entendiendo que la separación de lógica y matemática es un residuo histórico que es preciso eliminar por razones teóricas. Hay que señalar a Morgan, Boole, Bayues, Stanley, Jevons, Hilbert, 
Camap, García Bacca. La lógica pura es la iniciada por Bolzano, desarrollada por Husserl y defendida y expuesta por Pfänder [...]". Esta es toda la información que se daba sobre la logica matemática y las corrientes analíticas. El otro texto que quiero traer a colación, en relación al "espíritu de la época", pertenece a otro conocido manual que al filo de hablar de la materia y la forma dice así: «En los siglos XIX y $\mathrm{XX}$ se han desarrollado otros tipos de materialismo. Son los siguientes: el materialismo dialéctico, prolongación del anterior por obra de Carlos Marx y de los numerosos marxistas que a su ve $z$ han derivado a través del socialismo en el materialismo comunista o soviético, sucesivamente interpretado e impuesto por sus dos jefes supremos Wladimir Ilpitsch Ulianov (Lenin) y José Vsarionovitsch Djugosvili (Stalin); el materialismo científico en alianza con el positivismo y en contacto con las ciencias naturales representado por Jacobo Molschott, Carlos Vogt.... (y una lista de personajes que no han tenido la suerte histórica del Sr. Düring). ¿Merecen algín comentario estos textos? Pienso que por sí mismos dan una muestra del estilo de los dirigentes filosóficos (académicos) de la época.

También es cierto, hay que señalarlo, que no todo el panorama era tan sombrío. Carlos París había publicado en 1952 Física y filosofí; en ésta como en otras de sus obras el tratamiento del pensamiento neopositivista era desde el legítimo distanciamiento crítico, serio e inusual en aquellas épocas; igualmente los profesores E, Lledó, F. Montero, entre otros, dialogaban abiertamente con el pensamiento analítico.

\section{La recepción de la filosofía analitica}

Historiar este movimiento, que en el fondo forma parte del periplo indivi- dual de muchos de los estudiantes y profesores de aquellas generaciones, es tarea agradable, pero siempre que se tome con la mejor buena voluntad, sin que se exija un distanciamiento que, al menos a mí, no me es posibie.

Dos factores conjuntados pueden explicar el proceso, por un lado la apertura editorial y por otro la maduración de la conciencia universitaria que comienza a hacer frente a la filosofia ( $y$ a la ideología) oficial. Por lo que respecta a la apertura editorial hay que destacar la publicación en 1957, en Revista de Occidente, de la traducción de Tierno Galván del Tractatus Logico-Philosophicus, y la publicación un año después en la misma editorial de la traducción de Montserrat Macau de Lledó de La revolución en filosofia. Ambas obras tardaron en influir y ser punto de referencia en los estudios de la filosofía, pero a la larga cumplieron su papel. La primera, el Tractatus, ponía al alcance de la joven filosofia española una obra gigante de la filosofía analítica clásica; la otra nos informaba de un nuevo movimiento, la llamada filosofía analítica del lenguaje ordinario, gestado en la Inglaterra de los años cincuenta, a raíz, como es sabido, de una segunda obra de Wittgenstein que ha tardado demasiados años en ver la luz en las lenguas peninsulares. Estas obras junto con el incremento de producción bibliográfica, tanto de traducciones como de producciones propias en las décadas de los sesenta y setenta marcan uno de los polos de la recepción de la filosofía analítica.

El otro factor al que aludía consiste en la concreción de un período de gran vitalidad en la universidad española: una lucha de los universitarios contra el régimen dictatorial unida a um sincero deseo de renovación cultural e ideológica. En este contexto, la filosofía analítica representaba un aire renova- 
dor, crítico y riguroso frente a la filosofía oficial de la Academia y del régimen. El movimiento universitario, estudiantes y jóvenes profesores (conviene advertir que esta situación, como es lógico, coincide con el inicio del proceso de incremento del acceso a la universidad debido al desarrollismo económico, y ello provoca un incremento de jóvenes profesores que van tomando cucrpo y peso en la vieja estructura aristocrática de la Universidad) necesitábamos alternativas a aquel espíritu de la época al que me he referido en párrafos anteriores.

\section{La recepción politica: analítica y dialécrica}

Pero en este contexto la función de la filosofía analítica fue un tanto esquizofrénica: buena parte de estudiantes $y$ jóvenes profesores se veían inmersos en la alternativa entre una fundamentación teórica en el materialismo dialéctico de su práctica política habitual y un respeto honesto hacia la lógica matcmatica, la filosofía de la ciencia y la filosofía analítica en todas sus vertientes, ya que suponían un potente instrumental critico para desmontar las pseudoconstrucciones teóricas oficialistas. Esa esquizofrenia marcó toda esa época (la segunda mitad del sesenta y la primera del setenta, es decir, aproximadamente del sesenta y cinco al setenta y cinco, fecha de la muerte de Franco) y me atrevo a decir que hubo una coexistencia pacífica entre marxistas y francfortianos; los primeros respetaban, aunque tachaban de pequeño-burgueses y tecnocratas, a los analíticos, y entre analíti$\cos$, aunque en privado confesaron que el diamat no daba teóricamente para mucho, se respetaba a los marxistas.

Eran harto frecuentes en la época los coloquios y mesas redondas para aclarar el tema de la compatibilidad entre analítica y dialéctica, a ello podrán contribuir figuras como la de Manuel Sacristán, pensador marxista y a la vez introductor de la lógica matemática, traductor de importantes obras de Quine y propulsor (en Sobre el lloc de la filosofia en els estudis superiors, Barcelona, 1968) de posiciones epistemológicas, "la filosofía como saber adjetivo", próximas al positivismo lógico; pero no sólo la figura de Manuel Sacristán, que cito por ser la más señera, su posición «espiritual» era compartida por buena parte de profesores y estudiantes de filosofía.

En el año sesenta y cinco, Ferrater Mora publica La filosofia en el món d'avui, que es la versión catalana de su libro Philosophy today, y que nos informa, aparte de otras cuestiones, del estado de la filosofía en Europa y Améri$c a, y$ con ello se nos presenta una visión global de las alternativas sistemáticas, epistemológicas y metodológicas de la filosofía xen el món d'avuis. Ese mismo ano aparece en FCE la traducción de la recopilación de Ayer. El Positivismo lógico; en ella se nos daba a conocer textos clave del Circulo de Viena y textos de la nueva filosofía analítica.

$\mathrm{Ni}$ que decir tiene que en la misma época se recibía amplia producción filosófica del llamado materialismo dialéctico que fue conformando también un estilo de pensamiento y una sensibilidad para cuestiones sociopolíticas, históricas, ideológicas, etc., a las que la filosofía analítica mostraba poca sensibilidad. Creo, sin embargo, que el clima fue, en general, bastante tolerante entre ambas sensibilidades. Javier Muguerza vio con ojos benignos (y certeros) esa mutua tolerancia cuando escribió en «Esplendor y miseria del análisis filosófico» lo siguiente: "Junto a esta comunidad de intereses teóricos [...], pudieran también darse coincidencias de orden práctico entre análisis filosófi- 
co y marxismo celtibéricos: quien no desee hacer suyas las previsiones antes apuntadas en materia de política cultural podría considerarlos, por ejemplo, como aliados potenciales frente al enemigo común que es la filosofía oficial residual. $Y$, como alguna vez he recordado, entre ambos cabría incluso la posibilidad de alguna suerte de complementariedad funcional: el complemento analítico podría contribuir a aliviar de su carga dogmática al marxismo, lo que sería de celebrar puesto que un dogma es cosa muy distinta de un pensamiento vivo; el complemento marxista, por su lado, podría impedir entre otras cosas que la filosofía analítica llegara a convertirse en la nueva filosofía oficial de una futura España tecnocrática como la que seguramente se nos avecinam. ${ }^{3}$ Esto lo escribe Muguerza en el año 1974, dieciséis años después habrá de reconocer conmigo, que la filosofía analítica no se ha convertido en filosofia oficial, y no precisamente gracias al marxismo (a no ser que se entienda que el hecho de que el vicepresidente del Gobierno felicite las Navidades con un conocido texto de Bertrand Russell constituya una oficialización de la filosofia analítica).

Ese espíritu de colaboración es el que animó a Alfredo Deaño a preparar un número monográfico de Revista de Occidente ( $\mathrm{n}^{\circ} 138$, septiembre 1974) bajo el título Análisis y Dialéctica. Deaño no pretendió en ese número una confrontación de métodos y filosofias demasiado tópica en aquel momento, sino una colaboración constructiva, que cada uno dijera la suya sobre problemas concretos; así lo expresa el propio Deaño en su estudio de presentación y justificación del número: adel problema al que nos hemos asomado en este trabajo introductorio darán ellos (los colaboradores: $X$. Rubert de Ventós, Vidal Peña, J. Muguerza,
J.L. Blasco y M.A. Quintanilla) cuenta de la única manera posible: en concreto. Sólo a través del examen de problemas específicos -éticos, estéticos, metafísicos, epistemológicos, de filosofía política- será posible - y a ello queríamos ayudar con este número de Revista de Occidente- convertir el problema que llena el vago nombre de Análisis y Dialéctica en algo tan urgente y palpable como para que la filosofia - y, especialmente, los filósofos de nacionalidad española- se exijan un replanteamiento del mismo de manera cada vez más rigurosa, elaborada y plena”."

En este ambiente, que quizá no era tan idílico como aparece aquí reflejado, ya que habría sus críticas, réplicas y contrarréplicas de unos y otros, ${ }^{5}$ se desarrolló en la década de los setenta el momento de mayor auge de la filosofía analítica en nuestra comumidad filosófica.

\section{Et desarrollo de la filosofia analitica}

Muy sucintamente expondré ahora algunos (ya que estoy seguro de que omitiré sin querer) de los focos de la epidemia analítica. Madrid, Barcelona y Valencia constituyeron los núcleos más activos, lo cual era lógico ya que se trataba de los únicos distritos universitarios que contaban con estudios de filosofía, y si bien es cierto que las facultades de filosofía servían para poco (especialmente alguna), no menos cierto es que en esos distritos se concentraba el mayor número de estudiosos de la filosofía (aunque la tuvieran que estudiar al margen del alma mater).

En Madrid se tradujo y se produjo. Buena prueba de ello son los trabajos de Javier Muguerza, José Hierro, Víctor Sánchez de Zavala, Alfredo Deaño, y un largo etcétera. La contribución del grupo de Madrid a través de la actividad editorial (fundamentalmente Tecnos, Taurus, Alianza, Revista de Occidente y otras) su- 
pone la introducción en España de trabajos fundamentales de la tradición analítica (sirvan de ejemplo las traducciones y comentarios de Muguerza y Solís, de Russell y Moore, la publicación en 1962 de la traducción de Sánchez de Zavala de la Lógica de la investigación cientifica de Popper y muchos otros más).

Sin duda, la actividad editorial de introducir en España pensamiento europeo era ( $\mathrm{y}$ sigue siendo) importantísima después de que en 1939 se repitiera el cierre cultural de fronteras, que ya estableció Felipe $\Pi$, y a esa actividad nos dedicamos muchos de los "filósofos jóvenes" de los afios sesenta y setenta. Madrid disponía de mayores medios editoriales y a la vez de un grupo sólido para hacer frente a esta tarea.

Pero, por lo que a Madrid respecta, no se trata sólo de encomiar su labor editorial; es más importante la producción teórica de todo el plantel de lógica, filosofía de la ciencia y del lenguaje, que en su mayoría fuera de los ambientes académicos, $o$ dentro de ellos a contrapelo, desarrollaron la investigación «analítica» en este dichoso país. No hará falta recordar los trabajos de Muguerza (que, aunque le gusta distanciarse de la filosofía analítica, la cultivó, aunque heterodoxamente como todos) sobre mil y una cuestiones: Moore, Russell, falacia naturalista..., los estudios semióticos de Sánchez de Zavala y los trabajos sobre filosofía del lenguaje de José Hierro, así como la lógica de A. Dcan̆o (lamento dejame en el tintero muchas producciones importantes, pero trato de hacer una crónica de un período filosofico de nuestra historia y no un análisis exhaustivo de la producción analítica en nuestros lares).

Quiero hacer una mención especial en este contexto al proyecto frustrado de J. Muguerza Lecturas de filosofia analitica; cra un proyecto ambicioso de 5 volúmenes en Alianza Editorial, de los que sólo ha aparecido el primero (en dos tomos) y que prometía ser un instrumento bastante completo y útil para los estudiosos del tema. Especialmente lamento que no llegara a publicarse un volumen (el último: Balance y perspectiva de la filosofia analitica) que, según confesiones personales de J. Muguerza, pretendía incluir un elenco de la producción analítica en España; posiblemente la publicación de ese volumen hubicra hecho inútil cste escrito.

Un acontecimiento importante que es necesario mencionar en esta crónica fue el Simposio de Burgos, en 1968, que, organizado por Luis Martín Santos y Francisco Herman, congregó en torno a Popper a estudiosos de la epistemología, fueran filósofos o de otras disciplinas: la editorial Tecnos publicó en 1970 las actas y alli figura la lista de participantes, que omito repetir.

Terminaré esta referencia, escueta $y$ pobre, no lo dudo, a la producción madrileña, narrando que el "grupos de $\mathrm{Ma}$ drid, en su mayoría, pudo afortunadamente integrarse en la Academia con el traslado del profesor Carlos París desde la Universidad de Valencia a la Autónoma de Madrid. Allí se constituyó un departamento de filosofía en el que pudo reunirse un trabajo de muchos años.

En Barcelona también hubo, obviamente, actividad editorial sustantiva en la recepción de la filosofía analítica, editoriales como Ariel, Grijalbo, Edicions 62 , Labor, etc., con traductores y traducciones tan importantes como $\mathbf{M}$. Sacristán, Jacobo Muños, J. Mosterín... Sin embargo, la Universidad de Barcelona fue remisa en su contribución a la introducción de la filosofía analítica. Por un lado Manuel Sacristán, autor de la Introducción a la lógica y al análisis formal y traductor de importantes obras de Quine, estaba fucra de la Universidad por razones no por frecuentes en la época menos injustas, y alternaba 
su dedicación a la lógica y la filosofía científica con su firme compromiso ideológico con el pensamiento marxista.

En la Universidad, Jesús Mosterín desde sus inicios se dedicó al cultivo de la lógica formal tanto con sus obras (Lógica del primer orden y Teoría axiomática de conquistas) como con su docencia que hizo posible obras como La estructura del mundo sensible de Ulises y Moulines, y otros más. Hay que reconocer, sin embargo, que en aquellos años, la Facultad de Filosofía de Barcelona andaba lejos de estos parajes. Jesús Mosterín logró normalizar la lógica formal en la docencia y la investigación, y progresivamente fueron surgiendo investigadores y producciones en filosofía de la ciencia y filosofía del lenguaje. Daniel Quesada (La lingiiustica generativo transformacional: supuestos e implicaciones e Introducción a la filosofía del lenguaje, junto con J.J. Acero) y I.I. Acero (Filosofía y análisis del lenguaje, entre otras) son exponentes de unas líneas de investigación que han encajado en el Departamento de Lógica de Barcelona y en otras universidades como la de Granada.

En Valencia se dio la peculiar circunstancia de una pronta constitución, aunque sólo fuera de facto, de un Departamento de Lógica y Filosofía de la Ciencia, dirigido por Manuel Garrido. Además de la actividad traductora normal, quizá menos abundante ya que la riqueza editorial de Valencia es menor que la de Barcelona o Madrid, este Departamento desarrolló dos actividades especialmente relevantes en la recepción de la filosofía analítica. Los Simposia de lógica y filosofía de la ciencia y la revista Teorema.

Los Simposia se desarrollaron desde finales de los sesenta hasta finales de los setenta, si no me falla la memoria. Su inicio estuvo ligado a la lógica y filosofía analítica alemana y proporcio- naron la venida a Valencia de Hasenjaeger, Kutchera, Ch. Thiel y A. Diemer, entre otros. El tercer Simposio (1971) estuvo dedicado a una revisión de la filosofía y la ciencia en el pensamiento español de la década de los sesenta, y presidido por Ferrater Mora, en él coincidieron parte de los filósofos y científicos del momento; las actas, como es sabido, están publicadas en editorial Tecnos (Filosofía y Ciencia en el pensamiento español contemporáneo). Debo confesar, como colaborador cualificado, que organizar aquellos aaquelarres" filosóficos era toda una aventura. A partir del IV Simposio, estas reuniones se decantaron fundamentalmente a la filosofía analítica de corte anglosajón y aparecieron por Valencia pensadores como Strawson, Pears, Quine, etc. De todas ellas se tiene publicación.

La importancia de los Simposia radicó fundamentalmente en conocer by acquaintance a algunos de los protagonistas relevantes del pensamiento analítico, dialogar con ellos, escucharlos y que nos escucharan. Constituyeron, a mi juicio, una importante ruptura de fronteras.

La otra actividad importante del Departamento de Valencia fue la revista Teorema. Esta revista nació en marzo de 1971 con una vocación renovadora en el panorama filosófico español, en el sentido de integrar todas las dimensiones del pensamiento contemporáneo. Así en la presentación del primer número los profesores M. Garrido y F. Montero (codirectores de la revista) afimaban «Teorema intenta recoger y estimular aquellas investigaciones que versen sobre problemas filosíficos modernos abordados desde un punto de vista crítico. Bajo esta perspectiva, cualesquiera temas y problemas acordes con el actual momento filosófico, desde la lógica matemática y la filosofía de la ciencia y de la técnica a la dialéctica, 
pasando por la fenomenología y el estructuralismo tienen cabida en estas páginas. La publicación de Teorema, que se inserta en una linea de tan escasa tradición en la filosofía española, responde, creemos, a una necesidad que se agudizaba día a día en amplios sectores del quehacer filosófico peninsular'.

Con este espíritu nació Teorema, publicada conjuntamente por los Departamentos de Lógica y Filosofía de la Ciencia y de Historia de la Filosolia, codirigida por los profesores Garrido y Montero. Así, el primer número se presentaba bajo el tema «Razon analítica y razón dialéctica».

Este espíritu omnicomprensivo desapareció al año siguiente y la revista pasó a ser exclusiva del Departamento de Lógica y dirigida por M. Garrido. Las razones de ese cambio son obvias: la revista desde el principio nacio escorada hacia la lógica, la filosofía de la ciencia y el pensamiento analítico; aunque esta vocación omniabarcante que a veces le daba un pretendido carácter de suma cognitionum, la mantuvo siempre. La revista sirvió no sólo ni principalmente para publicaciones de los investigadores españoles sino tambièn para introducir originales o traducciones de pensadores europeos o americanos. Teorema vivió en Valencia hasta el año 1980, en el 1981 se trasladó a Madrid e hizo realidad una vez más el dicho castizo "de Madrid al Cielon.

Al lado de la revista, en su etapa valenciana, se desarrolló una actividad importante: los números monográficos que respondían a los simposia de los que antes he hablado y los Cuademos teorema, que han introducido en lengua castellana monográficas importantes, clásicas o del momento.

Por lo demás, la producción filosófica del grupo de Valencia fue el fruto estimable de la actividad de un depar- tamento que dentro de sus contradicciones permitió la formación de un grupo, de tendencias dispersas, hoy repartidas por la geografia universitaria peninsular. Citaré sólo, a modo de ejemplo y con la rigurosa petición de perdón a todos mis compañeros mi Lenguaje, Fitosofia y Conocimiento (Barcelona, Ariel, 1973), la Lógica Simbólica de Manuel Garrido (Madrid, Tecnos, 1976), y los trabajos de Rafael Beneyto y J. Sanmartín, entre otros.

No quiero acabar este epígrafe sin reconocer que muchos otros estudiosos de la filosofía, fuera de los centros universitarios que he considerado, tuvieron una importante función en la recepción del análisis filosófico; es necesario mencionar a Vicente Muñoz Delgado que dedicó una seria investigación a la historia de la lógica y el pensamiento analítico clásico, y en la misma Salamanca M.A. Quintanilla se dedicaba a la filosofía de la ciencia y publicó en 1972, en Tecnos, uno de los primeros estudios sobre Popper: Idealismo y filosofía de la Ciencia.

\section{Balance}

¿Cuál ha sido el resultado de tanto esfuerzo editorial, de ruptura de fronteras, de investigación, etc.? No dudo que el sarampión analítico de los años sesenta y setenta ha sido beneficioso en nuestro panorama filosófico actual (como tambiến lo han sido otros sarampiones de los que no me correspondía hablar), pero la filosofía española (?) sigue siendo una filosofía de recepción, lo cual, dicho sea en honor a la verdad, es mucho mejor que cerrar las fronteras.

Por supuesto que nadie defiende hoy (al menos que yo sepa) ni las tesis del positivismo lógico, ni las de Russell, ni el Tractatus, ni la idea de que la filosofía es enseñar a la mosca el camino de 
salida de la botella, pero como dice Mosterín en un texto que algunos aceptariamos: "casi nadie acepta hoy en día las tesis sustantivas de la filosofia analitica clásica. Pero casi todos hemos aprendido y heredado algo de ella, aunque no sea más que un cierto talante, unos ciertos standares, unas ciertas maneras, una cierta transparencia en el pensar y en el hablac». ${ }^{6}$

El método y sobre todo el «estilo» analítico de filosofar (por lo demás tan antiguo como Aristóteles, Tomás de Aquino, Kant, etc.) están sólidamente asentados en los departamentos de lógica y no me refiero a la lógica misma cuya naturaleza ya nadie discute, sino a disciplinas como la filosofía de la ciencia y la filosofía del lenguaje, en estos ămbitos la investigación sigue los derroteros, heterodoxos siempre (eso es una premisa de todo pensar filosófico), del "espíritu» $\mathrm{y}$ "método" analíticos.

En el área de la ética hubo unos inicios de incorporación metodológica: el temprano libro de J. Hierro: Problemas del análisis del lenguaje moral (Madrid, Tecnos, 1970), el de Victoria Camps:
Pragmática del lenguaje y filosofía analítica (Barcelona, Península, 1976), y otros trabajos. Sin embargo no se ha asentado el análisis en los estudios éticos de muestros lares. $\mathrm{Y}$ ello tiene una razón: hay que reconocer que el proceder analítico no ha generado, aparte del incipiente discurso sobre el razonar ético, rejas relevantes de análisis e investigación sobre problemas morales.

Y por lo que respecta a la hace tiempo llamada filosofía teorética, he de reconocer que el impacto de la filosofía analítica en ella ha sido prácticamente nulo. A excepción de la teoría del conocimiento (o epistemología) que se hace en Valencia, que es en general de ucorten analítico (o si se prefiere anglosajón), el resto es más deudor de una recepción de la filosofía germánica ( $\mathrm{He}$ gel, Hcidegger...).

Terminaré diciendo que la filosofía no es una cuestión de escuchas ni menos de banderias: los métodos y los problemas han de ser intercambiables y hay que volver al agora donde todos dialoguemos y seamos conscientes de que hablamos sobre lo mismo.
1. No voy a intentar en estas páginas presenlar la nómina de todos los que de una forma u otra. han contribuido a la introducción de la filosofia analitica en muestro panorama filosofico. Quiero decir con ello que mí punto de vista será global sobre el impacto de la filosofia analítica en un momento crucial de la universidad española, al menos por lo que a la filosofía se refiere. Por ello pido de antemano disculpas por todas las omisiones, sin duda importantes en todos los casos, que pueda contener.

2. Y los profesores más osados que comencaban a prepara la apertura de los años sesenta.

3. Javier Muguerza: *Esplendor y miseria del análisis filosóficow en J. Muguerza (ed.) $T_{a}$ Con- cepcion analitica de la flosofia, I, Madrid, Alianza Editorial, 1974, p. 131.

4. A. Deaño, "Análisis y dialéctica: la razón de unas páginas*, Revista de Occidente, 138, septiembre (1974), p. 149.

5. Yo mismo en el artículo de Revista de Oct: dente en al mencionado número hablaba de que dedicarse a la filosofía analítica no fuera mmerecedor de las penas del infierno especialmente destinado a los filosofos analíticos, ese infierno de escolasticismo y tocnocracia que, con lanto afăn, nos están creando algunos terrorificos jueces: (p. 236). (Y por supuesto que me referia a jueces concretos!)

6. J. Mosterin (prólogo a J.J. Acerol, Filosofia y Andisis del leriguaje, Madrid, Cincel, 1985, p. 15. 